\section{WIDESPREAD EXTRA-MOTOR ABNORMALITY IS A PROMINENT MRI SIGNATURE OF ALS: A CROSS- COHORT STUDY}

${ }^{2,1}$ Sicong Tu*, ${ }^{2}$ Martin R Turner, ${ }^{1}$ Matthew C Kiernan. ${ }^{1}$ Brain and Mind Centre, University of Sydney, Sydney, NSW, Australia; ${ }^{2}$ Nuffield Department of Clinical Neurosciences, University of Oxford, Oxford, UK

\subsection{6/jnnp-2019-anzan.17}

Introduction Amyotrophic lateral sclerosis (ALS) is a heterogeneous neurodegenerative disease characterised by motor dysfunction, but now recognised as a complex multi-system disorder. Neuroimaging studies' indicate an expanding, though inconsistent, list of extra-motor neural involvement. The objective of the current study was to examine pattern of grey matter change across two clinically well matched patient cohorts to identify core neural changes underlying ALS.

Methods Independent ALS and age-matched healthy control cohorts were compared from Oxford (ALS: 45; Control: 34) and Sydney (ALS: 45; Control: 27). Whole-brain voxel-based morphometry and subcortical volumetric analyses were carried out.

Results Subcortical volumetric reduction was consistently observed in the thalamus, caudate and hippocampus (all p < 0.05). Pattern of cortical grey matter atrophy showed variability across ALS cohorts, but consistently implicated crus I-II of the cerebellum and orbitofrontal cortices $(\mathrm{p}<0.01$, familywise error corrected).

Conclusions The core grey matter MRI signature of ALS comprises extensive extra-motor cortical and subcortical abnormality. Significant variability exists, which highlights the heterogeneous nature of ALS as defined by current diagnostic guidelines.

\section{ROLE OF TRANSCALLOSAL INHIBITION IN DISEASE SPREAD IN ALS}

3,1,2Mehdi Van den Bos*, ${ }^{4}$ James Howells, ${ }^{2}$ Mana Higashihara, ${ }^{3,1,2}$ Nimeshan Geevasinga, ${ }^{4}$ Matthew Kiernan, ${ }^{3,1,2}$ Steve Vucic. 'University of Sydney, Sydney, NSW, Australia; ${ }^{2}$ Department of Neurology, Westmead Hospital, Sydney, NSW, Australia; ${ }^{3}$ Westmead Institute, Westmead Hospital, Westmead, NSW, Australia; ${ }^{4}$ Brain and Mind Centre, University of Sydney, Sydney, NSW, Australia

\subsection{6/jnnp-2019-anzan.18}

Introduction There is substantial evidence for the cortical origin of amyotrophic lateral sclerosis (ALS). Impaired function of the corpus callosum has been demonstrated in ALS patients and may play a role in disease spread, potentially mediating the spread of cortical hyperexcitability between hemispheres. We assessed transcallosal inhibition, utilising the threshold tracking transcranial magnetic stimulation (TMS) technique to assess transcallosal inhibition and related the changes to disease involvement.

Methods Threshold tracking TMS was undertaken in 15 ALS patients and results were compared to 16 healthy controls. Interhemispheric inhibition was assessed using a figure of eight coil over each hemisphere across interstimulus intervals ranging from 8 to $40 \mathrm{~ms}$.

Results Transcallosal inhibition was reduced in ALS patients $(0.9 \pm 1.0 \%)$ when compared to controls $(6.6 \pm 1.0 \%$, $\mathrm{P}=0.03)$. Importantly differences in transcallosal inhibition between hemispheres were evident in ALS patients.
Specifically, transcallosal inhibition projecting from the motor cortex contralateral to disease onset $(0.9 \pm 1.0 \%)$ was significantly lower when compared to projection form the ipsilateral motor cortex (3.2 $\pm 1.0 \%, \mathrm{P}=0.036)$. Abnormalities of transcallosal inhibition correlated with upper motor neurone dysfunction and greater functional disability in ALS.

Conclusion Abnormalities of transcallosal inhibition were demonstrated in ALS patients and were associated with clinical features. Consequently, dysfunction of transcallosal fibres may contribute to development of cortical hyperexcitability, a pathogenic mechanism in ALS. Strategies aimed at modulating dysfunction may prove therapeutically useful in ALS.

\section{INCREASED RISK OF AN ABNORMAL CERVICAL SCREENING TEST IN WOMEN WITH MS EXPOSED TO HIGH-EFFICACY DISEASE-MODIFYING TREATMENTS}

${ }^{4,1,2,3}$ Anneke Van der Walt*, ${ }^{1}$ Jeremy Yuvaraj, ${ }^{1}$ Jim Stankovich, ${ }^{4}$ Nicola McGuinn, ${ }^{4}$ Louise Rath, ${ }^{4}$ Olga Skibina, ${ }^{4}$ Cassie Nesbitt, ${ }^{4,1}$ Robb Wesselingh, ${ }^{4,1,2}$ Mastura Monif, ${ }^{4}$ Janene Richards, ${ }^{2,3}$ Tomas Kalincik, ${ }^{2}$ Lisa Taylor, ${ }^{2}$ Josephine Baker, ${ }^{2,3}$ Ai-Lan Nguyen, ${ }^{5}$ David C Wrede, ${ }^{4,1}$ Helmut Butzkueven, ${ }^{7,6}$ Julia Brotherton, ${ }^{4,1}$ Vilija Jokubaitis. ${ }^{1}$ Neuroscience, Central Clinical School, Monash University, Melbourne, VIC, Australia; ${ }^{2}$ Neurology, Royal Melbourne Hospital, Melbourne, VIC, Australia; ${ }^{3}$ Department of Medicine, RMH, Melbourne Brain Centre, University of Melbourne, Melbourne, VIC, Australia; ${ }^{4}$ Alfred Health, Melbourne, VIC, Australia; ${ }^{5}$ Cervical Dysplasia Service, Royal Women's Hospital, Melbourne, VIC, Australia; ${ }^{6}$ VCS Population Health, VCS Foundation, Melbourne, VIC, Australia; 'Victorian Cenvical Screening Registry, Melbourne, VIC, Australia

10.1136/jnnp-2019-anzan. 19

Introduction Long-term exposure of women with Multiple sclerosis (MS, wwMS) to immunomodulatory or immunosuppressive treatments may increase the risk of cervical dysplasia. However, little is known about cervical dysplasia risk and Human Papillomavirus (HPV)-vaccine coverage in wwMS.

Methods Adult wwMS were recruited from two tertiary MS clinics. To explore the association between MS treatments (DMTs) and abnormal cervical screening tests (CSTs), we linked individual data from MSBase, the Victorian Cervical Screening Registry, and National HPV vaccination program registry (NHPVPR).

Results To date, we have recruited 208 wwMS of whom 102 had complete data (vaccination status, cervical screening tests, MSBase data) and no previous history of abnormal CST at MS onset for this interim analysis. The average age was 33.8 (18 to 59 yrs) and most $(n=58,88 \%)$ were unvaccinated. 19 wwMS (19\%) had an abnormal CST after MS onset (incidence rate 20.6 cases $/ 1000$ person-years, 95\% confidence interval 12.4-32.1) over average 9.0 years of follow-up. 57 wwMS were treated with lower-efficacy therapies (56\%), 73 with a high-efficacy therapy (72\%), and 44 were exposed to both. Eight abnormal CSTs were detected before starting high-efficacy therapy (rate 12.6, 95\% CI (5.4-24.8)) and 11 were detected after starting high-efficacy therapy (rate 38.6, 95\% CI (19.3-69.0), $\mathrm{p}=0.022$.

Conclusion We provide preliminary data that high efficacy DMTs may increase the risk of abnormal CSTs over time. A larger cohort and inclusion of additional cervical dysplasia risk factors are required to fully elucidate risk in wwMS. 